\title{
The Riemann Integral Construction of The Sequence of Functions in a Normed Space $\left(\ell^{p},\|\cdot\|_{p}\right)$
}

\author{
Aswad Hariri Mangalaeng ${ }^{1 *}$, Naimah Aris ${ }^{1 *}$, Jusmawati Massalesse $^{1 *}$
}

\begin{abstract}
We construct Riemann Integral for a sequence in a normed space $\left(\ell^{p},\|\cdot\|_{p}\right)$. To do construction, we used some theory of real analysis and functional analysis, include some real sequences theory, some Riemann integral theory for functions in $\mathbb{R}$, and some norm theory in a normed space $\left(\ell^{p},\|\cdot\|_{p}\right)$. On this research, we otained that a sequence of functions $\boldsymbol{f}=$ $\left(f_{k}\right):[a, b] \subset \mathbb{R} \rightarrow \ell^{p}$ qualify that the sequence is Riemann integrable on $[a, b] \subset \mathbb{R}$.
\end{abstract}

Keywords: Sequence of functions, Riemann integral construction, Normed space $\ell^{p}$.

\section{Introduction}

Integrals are one of the important concepts in mathematical analysis that develops very well. Its development began in the late 17th century and to date has been a lot of research that produces the latest theories and the various applications of integrals. The integral concept was first put forward by two mathematicians, namely ISAAC Newton in the late 1660s and Gottfried Leibniz in the 1680s. Both found that integrals were the opposite of derivatives. For a century and a half after this concept remained in use. Later in 1850-an German mathematician (Georg Friedrich) Bernhard Riemann introduced a new and different viewpoint. Bernhard Riemann discovers that the concept of integrals and derivatives can be separated.

In the concept found by Bernhard Riemann, an integral of a function in the domain in the form of closed and limited intervals $[a, b] \subset \mathbb{R}$ can be defined without the use of derivatives, which is preceded by partitioning at intervals $[a, b] \subset \mathbb{R}$, then defining integral value of the function as the limit of Riemann sum. The integral concept made by Bernard Riemann is known as the Riemann integral theory.

The study of the Riemann integral theory has been widely examined by mathematicians and many have successfully used it to define the integrals of a function, including the study conducted by Purnomo Aji [1] to construct Riemann integral of an sequence of functions in space $\ell^{1}$, namely by using the Riemann integral theory for functions in $\mathbb{R}$ that is obtained the sequence of functions in the space $\ell^{1}$ is Riemann integrable on $[a, b] \subset \mathbb{R}$.

Space $\ell^{1}$ is one of the sequences spaces in mathematical analysis. The general space of the space $\ell^{1}$ is space $\ell^{p}$, which is the space that contains all the sequences $\left(x_{n}\right)$ in the $\mathbb{R}$ so that the

\footnotetext{
${ }^{*}$ Department of Mathematics, Faculty Mathematics and Natural Sciences, Hasanuddin University

Email: 'aswadh2905@gmail.com, ${ }^{1}$ newima@gmail.com, ${ }^{1}$ jusmawati@gmail.com
} 


\section{Aswad Hariri Mangalaeng, Naimah Aris, Jusmawati Massalesse}

series $\sum_{n=1}^{\infty}\left|x_{n}\right|^{p}$ is convergence for each real number $p$ with $1 \leq p<\infty$. Space $\ell^{p}$ is said to be a vector space if the space fulfills vector space properties. Vector space $\ell^{p}$ that defined the norm inside is called a normed space. Norm on $\ell^{p}$ is a mapping of $\|\cdot\|: \ell^{p} \rightarrow \mathbb{R}$ that meets the following properties, for each $\boldsymbol{x}=\left(x_{n}\right), \boldsymbol{y}=\left(y_{n}\right) \in \ell^{p}$ and $\alpha \in \mathbb{R}$,

(i) $\quad\|x\| \geq 0$.

(ii) $\quad\|x\|=0$ if and only if $\boldsymbol{x}=\mathbf{0}$.

(iii) $\quad\|\alpha \boldsymbol{x}\|=\alpha\|\boldsymbol{x}\|$.

(iv) $\quad\|x+y\| \leq\|x\|+\|y\|$.

A space $\ell^{p}$ is a normed space towards the norm $\|\cdot\|_{p}$, which is

for every $\boldsymbol{x}=\left(x_{n}\right) \in \ell^{p}[4]$

$$
\|x\|_{p}=\left(\sum_{n=1}^{\infty}\left|x_{n}\right|^{p}\right)^{\frac{1}{p}}
$$

Riemann integral construction for the functions mapping in a normed space was discussed by Edward Chernysh [3] in his writings "the Riemann integral For Functions Mapping To Banach Spaces". According to Chernysh, the construction is done by determining the Riemann sum of a function $\boldsymbol{f}:[a, b] \subset \mathbb{R} \rightarrow X(X$ is a normed space) and defining the requirements of the function is Riemann integrable on $[a, b] \subset \mathbb{R}$.

Based on the description above, the author feels interested to review how the construction of Riemann integral functions in the normed space $\left(\ell^{p},\|\cdot\|_{p}\right)$ with the aim of the research is to construct the Riemann integral of the sequence of functions $\boldsymbol{f}=\left(f \cdot{ }_{k}\right):[a, b] \subset \mathbb{R} \rightarrow \ell^{p}$, where $\ell^{p}$ is a normed space with norms defined by $\|\cdot\|_{p}$.

\section{Bibliography}

\subsection{Riemann Integral in $\mathbb{R}$ \\ Definition 2.1.1 [2]}

Let $I=[a, b]$ be a closed interval in $\mathbb{R}$. Then a partition of $I$ is a finite set $\mathcal{P}=\left\{x_{0}, x_{1}, \ldots, x_{n}\right\}$ of points in I such that

$$
a<x_{0}<x_{1}<\cdots<x_{n-1}<x_{n}=b .
$$

The points in $\mathcal{P}$ are used to divide $I=[a, b]$ into subintervals

$$
I_{1}=\left[x_{0}, x_{1}\right], I_{2}=\left[x_{1}, x_{2}\right], \ldots, I_{n}=\left[x_{n-1}, x_{n}\right] .
$$

The partition $\mathcal{P}$ is often also denoted by $\mathcal{P}=\left\{\left[x_{i-1}, x_{i}\right]_{i=1}^{n}\right\}$

\section{Definition 2.1.2 [2]}

Let $I=[a, b]$ be a closed interval in $\mathbb{R}$. If a point $t_{i}$ is chosen from each subinterval $I_{i}=$ $\left[x_{i-1}, x_{i}\right]$, for $i=1.2, \ldots, n$, then point $t_{i}$ is called the sample point of the subinterval $I_{i}$. A set

$$
\dot{\mathcal{P}}=\left\{\left[x_{i-1}, x_{i}\right], t_{i}\right\}_{i=1}^{n}
$$

from subintervals that corresponding sample points are called a tagged partition of $I$.

Definition 2.1.3 [2]

Let $\mathcal{P}$ be a partition of a closed interval $I=[a, b]$ in $\mathbb{R}$. The norm or mesh of $\mathcal{P}$ is a number

$$
\|\mathcal{P}\|=\max \left\{\left|x_{i-1}-x_{i}\right|\right\}_{i=1}^{n} .
$$

The norm of a partition is the length of the largest subinterval by which the partition divides interval $[a, b]$. 


\section{Aswad Hariri Mangalaeng, Naimah Aris, Jusmawati Massalesse}

To simplify the writing of this article, the author uses the tagged partition norm $\|\dot{\mathcal{P}}\|$ versus norm partition $\|\mathcal{P}\|$. This is because $\|\dot{\mathcal{P}}\|=\|\mathcal{P}\|$.

Definition 2.1.4 [2]

Let $\dot{\mathcal{P}}$ be a tagged partition of a closed interval $I=[a, b]$. The Riemann sum of a function $f:[a, b] \rightarrow \mathbb{R}$ that corresponds to $\dot{\mathcal{P}}$ is

\section{Definition 2.1.5 [2]}

$$
S(f ; \dot{\mathcal{P}})=\sum_{i=1}^{n} f\left(t_{i}\right)\left(x_{i}-x_{i-1}\right) .
$$

A function.$:[a, b] \rightarrow \mathbb{R}$ is said to be Riemann integrable on $[a, b]$ if there is a number $L \in \mathbb{R}$ such that for each $\varepsilon>0$, there is $\delta>0$ such that if $\dot{\mathcal{P}}$ is any tagged partition of $[a, b]$ with $\|\dot{\mathcal{P}}\|<\delta$, then

$$
|S(f ; \dot{\mathcal{P}})-L|<\varepsilon
$$

Whether written $L=\int_{a}^{b}$ for $\int_{a}^{b} f(x) d x$. The set of all Riemann integrable functions in $[a, b]$ is denoted by $\mathcal{R}[a, b]$.

It should be noted that the sample point $t_{i} \in\left[x_{i-1}, x_{i}\right], i=1,2, \ldots, n$ is taken arbitrarily from any tagged partition $\dot{\mathcal{P}}=\left\{\left[x_{i-1}, x_{i}\right], t_{i}\right\}_{i=1}^{n}$ and the interval partition $[a, b]$ is as many as subintervals, with $n$ referring to $n$ where $n \in \mathbb{N}$ results in $n \rightarrow \infty$. So according to Definition 2.1.5, the function $f:[a, b] \rightarrow \mathbb{R}$ is Riemann integrable function on $[a, b]$ if and only if

$$
\lim _{\|\dot{\mathcal{P}}\| \rightarrow 0} S(f ; \dot{\mathcal{P}})=\lim _{\|\dot{\mathcal{P}}\| \rightarrow 0} S(f ; \dot{\mathcal{P}})=L \text {. }
$$

\section{Theorem 2.1.1 [2]}

If $f \in \mathcal{R}[a, b]$, then the integral value of $f$ is uniquely determined.

2.2 Normed space $\left(\ell^{p},\|\cdot\|_{p}\right)$

\section{Definition 2.2.1 [4]}

Space $\ell^{p}$ is a space that contains all sequences $\left(x_{n}\right)$ in the field $\mathbb{R}$ such that the sequence $\sum_{n=1}^{\infty}\left|x_{n}\right|^{p}$ is convergent for each real number $p$ to $1 \leq p<\infty$. Written :

$$
\ell^{p}=\left\{\boldsymbol{x}=\left(x_{n}\right) \in \omega: \sum_{n=1}^{\infty}\left|x_{n}\right|^{p}<\infty\right\}
$$

where $\omega$ is a collection of all sequences of real numbers.

\section{Lemma 2.2.1 [4]}

Space $\ell^{p}$ with the norm function $\|\cdot\|_{p}: \ell^{p} \rightarrow \mathbb{R}$ is defined as follows:

$$
\|x\|_{p}=\left(\sum_{n=1}^{\infty}\left|x_{n}\right|^{p}\right)^{\frac{1}{p}}
$$

for every $\boldsymbol{x}=\left(x_{n}\right) \in \ell^{p}$. Thus, $\left(\ell^{p},\|\cdot\|_{p}\right)$ is a normed space.

\section{Riemann Integral Construction}

In this section, it will be showed Riemann integral construction on an sequence of functions in the norm space $\left(\ell^{p},\|\cdot\|_{p}\right)$ which is the sequence of functions $\boldsymbol{f}=\left(f_{k}\right):[a, b] \subset \mathbb{R} \rightarrow \ell^{p}$. The 


\section{Aswad Hariri Mangalaeng, Naimah Aris, Jusmawati Massalesse}

construction is similar to Riemann integral construction of real value functions, which is by defining integrals as limit of Riemann sum. Then, to determine the Riemann sum the concept of partitions $\mathcal{P}$ is used tagged partitions $\dot{\mathcal{P}}$ and norm of tagged partitions defined $\|\dot{\mathcal{P}}\|$ that defined on Definition 2.1.3, Definition 2.1.4, and Definitions 2.1.5. The next will be defined the Riemann summ of the sequence of functions $\boldsymbol{f}=\left(f_{k}\right):[a, b] \subset \mathbb{R} \rightarrow \ell^{p}$ and the requirements of the sequence to be Reimann integrable on $[a, b] \subset \mathbb{R}$.

Lemma 3.1 Let $\left(\ell^{p},\|\cdot\|_{p}\right)$ is a normed space. The Riemann sum of the sequence of functions $\boldsymbol{f}=\left(f_{k}\right):[a, b] \subset \mathbb{R} \rightarrow \ell^{p}$ over a tagged partition $\dot{\mathcal{P}}$ of $[a, b] \subset \mathbb{R}$ is

\section{Proof:}

$$
S(\boldsymbol{f} ; \dot{\mathcal{P}})=\sum_{i=1}^{n}\left(x_{i}-x_{i-1}\right)\left(t_{i}\right)
$$

It is known that the sequence of functions of $\boldsymbol{f}=\left(f_{k}\right) \in \ell^{\mathrm{p}}$ is the sequence of real functions $f_{k}:[\mathrm{a}, \mathrm{b}] \subset \mathbb{R} \rightarrow \mathbb{R}, k=1,2, \ldots$

So that it can be defined the Riemann sum of sequence of functions $\boldsymbol{f}$ over a tagged partition $\dot{\mathcal{P}}$ of $[a, b] \subset \mathbb{R}$ is the sequences of the Riemann integrable real functions $f_{k}:[\mathrm{a}, \mathrm{b}] \subset \mathbb{R} \rightarrow \mathbb{R}, k=$ $1,2, \ldots$ over a partition tagged $\dot{\mathcal{P}}$, ie

$$
S(\boldsymbol{f} ; \dot{\mathcal{P}})=\left(S\left(f_{1} ; \dot{\mathcal{P}}\right), S\left(f_{2} ; \dot{\mathcal{P}}\right), \ldots\right)
$$

From Definition 2.1.4, the Riemann sum of the real functions $f_{k}$ over the tagged partition $\dot{\mathcal{P}}$ is $\mathrm{S}\left(f_{k} ; \dot{\mathcal{P}}\right)=\sum_{i=1}^{n}\left(x_{i}-x_{i-1}\right) f_{k}\left(t_{i}\right)$, for each $k=1,2, \ldots$. Substitution of the Riemann sum to Equation (10), obtained

$$
\begin{aligned}
\mathrm{S}(\boldsymbol{f} ; \dot{\mathcal{P}}) & =\left(\sum_{i=1}^{n}\left(x_{i}-x_{i-1}\right) f_{1}\left(t_{i}\right), \sum_{i=1}^{n}\left(x_{i}-x_{i-1}\right) f_{1}\left(t_{i}\right), \ldots\right) \\
& =\sum_{i=1}^{n}\left(x_{i}-x_{i-1}\right)\left(f_{1}\left(t_{i}\right), f_{2}\left(t_{i}\right), \ldots\right) \\
& =\sum_{i=1}^{n}\left(x_{i}-x_{i-1}\right) \boldsymbol{f} .\left(t_{i}\right) .
\end{aligned}
$$

Lemma 3.2 Let $\left(\ell^{p},\|\cdot\|_{p}\right)$ is a normed space. If the sequence of functions of $\boldsymbol{f}=\left(f_{k}\right):[a, b] \subset$ $\mathbb{R} \rightarrow \ell^{p}$ is Riemann integrable on $[a, b] \subset \mathbb{R}$, then there is a sequence function $\boldsymbol{Y}=\left(Y_{k}\right) \in \ell^{p}$ with the properties that for every $\varepsilon>0$, there is $\delta>0$ such that

$$
\|S(\boldsymbol{f} ; \dot{\mathcal{P}})-\boldsymbol{Y}\|_{p}=\left\|\sum_{i=1}^{n}\left(x_{i}-x_{i-1}\right) \boldsymbol{f}\left(t_{i}\right)-\boldsymbol{Y}\right\|_{p}=\left(\sum_{k=1}^{\infty}\left|\sum_{i=1}^{n}\left(x_{i}-x_{i-1}\right) f_{k}\left(t_{i}\right)-Y_{k}\right|^{p}\right)^{\frac{1}{p}}<\varepsilon
$$

for all partitions tagged $\dot{\mathcal{P}}$ of $[a, b] \subset \mathbb{R}$ with $\|\dot{\mathcal{P}}\|<\delta . \boldsymbol{Y}$ is the Riemann integral value of $\boldsymbol{f}$, written $\boldsymbol{Y}=\int_{a}^{b} \boldsymbol{f}$ or $\boldsymbol{Y}=\int_{a}^{b} \boldsymbol{f} d x$.

Proof:

Supposedly, the sequence of functions $\boldsymbol{f}=\left(f_{k}\right):[a, b] \subset \mathbb{R} \rightarrow \ell^{p}$ is Riemann integrable on $[a, b] \subset \mathbb{R}$, meaning that $\boldsymbol{f}$ is a sequence of real functions $f_{k}:[\mathrm{a}, \mathrm{b}] \subset \mathbb{R} \rightarrow \mathbb{R}, k=1,2, \ldots$ that is Riemann integrable on $[\mathrm{a}, \mathrm{b}] \subset \mathbb{R}$. So, for each function $f_{k}, k=1,2, \ldots$ there is a number $Y_{k} \in \mathbb{R}$ 


\section{Aswad Hariri Mangalaeng, Naimah Aris, Jusmawati Massalesse}

with the property that for every $\varepsilon>0$ there is $\delta_{k}>0$ such that if $\dot{\mathcal{P}}_{\mathrm{k}}=\left\{\left[x_{i-1}^{k}, x_{i}^{k}\right], t_{i}^{k}\right\}_{i=1}^{n}$ are any tagged partitions of $[a, b] \subset \mathbb{R}$ with $\left\|\dot{\mathcal{P}}_{\mathrm{k}}\right\|<\delta_{k}$, then

$$
\left|S\left(f_{k} ; \dot{\mathcal{P}}_{k}\right)-Y_{k}\right|=\left|\sum_{i=1}^{n}\left(x_{i}^{k}-x_{i-1}^{k}\right) f_{k}\left(t_{i}^{k}\right)-Y_{k}\right|<\varepsilon
$$

Supposedly $\delta=\min \left\{\delta_{k}\right\}_{k=1}^{\infty}$ and supposedly $\dot{\mathcal{P}}=\left\{\left[x_{i-1}, x_{i}\right], t_{i}\right\}_{i=1}^{n}$ are a tagged partition of $[a, b] \subset \mathbb{R}$ with $\|\dot{\mathcal{P}}\|<\delta$. Because $\|\dot{\mathcal{P}}\|<\delta \leq \delta_{k}$ is obtained

$$
\left|S\left(f_{k} ; \dot{\mathcal{P}}\right)-Y_{k}\right|=\left|\sum_{i=1}^{n}\left(x_{i}-x_{i-1}\right) f_{k}\left(t_{i}\right)-Y_{k}\right|<\varepsilon,
$$

for every $k=1,2, \ldots$

From the Inequation (3), because $\varepsilon>0$ was arbitrary, then for a real number $p$ with $1 \leq p<\infty$, it is obtained

$$
\begin{gathered}
\left|\sum_{i=1}^{n}\left(x_{i}-x_{i-1}\right) f_{k}\left(t_{i}\right)-Y_{k}\right|^{p}<\varepsilon^{p} \quad(k=1,2, \ldots) \\
\left|\sum_{i=1}^{n}\left(x_{i}-x_{i-1}\right) f_{1}\left(t_{i}\right)-Y_{1}\right|^{p}+\left|\sum_{i=1}^{n}\left(x_{i}-x_{i-1}\right) f_{2}\left(t_{i}\right)-Y_{2}\right|^{p}+\cdots<\varepsilon^{p}+\varepsilon^{p}+\cdots \\
\sum_{k=1}^{\infty}\left|\sum_{i=1}^{n}\left(x_{i}-x_{i-1}\right) f_{k}\left(t_{i}\right)-Y_{k}\right|^{p}<\varepsilon \\
\left(\sum_{k=1}^{\infty}\left|\sum_{i=1}^{n}\left(x_{i}-x_{i-1}\right) f_{k}\left(t_{i}\right)-Y_{k}\right|^{p}\right)^{\frac{1}{p}}<\varepsilon^{\frac{1}{p}} \\
\left(\sum_{k=1}^{\infty}\left|\sum_{i=1}^{n}\left(x_{i}-x_{i-1}\right) f_{k}\left(t_{i}\right)-Y_{k}\right|^{\frac{1}{p}}<\varepsilon .\right.
\end{gathered}
$$

In the Inequation (3), because inequation applies to any arbitrary $\varepsilon>0$, it is obtained

$$
\left|\sum_{i=1}^{n}\left(x_{i}-x_{i-1}\right) f_{k}\left(t_{i}\right)-Y_{k}\right|=0 \text { or } \sum_{i=1}^{n}\left(x_{i}-x_{i-1}\right) f_{k}\left(t_{i}\right)=Y_{k}
$$

for every $k=1,2, \ldots$

Notice that $\boldsymbol{f} \in \ell^{p}$, then based on the properties of the vector space $\ell^{p}$ is obtained

$S(\boldsymbol{f} ; \dot{\mathcal{P}})=\sum_{i=1}^{n}\left(x_{i}-x_{i-1}\right) f\left(t_{i}\right)=\left(\sum_{i=1}^{n}\left(x_{i}-x_{i-1}\right) f_{1}\left(t_{i}\right), \sum_{i=1}^{n}\left(x_{i}-x_{i-1}\right) f_{1}\left(t_{i}\right), \ldots\right) \in \ell^{p}$.

So if we define a sequence of functions $\boldsymbol{Y}=\left(Y_{k}\right)=\left(Y_{1}, Y_{2}, \ldots\right)$ that meets Inequation (5), we get $\boldsymbol{Y}=S(\boldsymbol{f} ; \dot{\mathcal{P}})$. As a result of $(6)$ obtained $\boldsymbol{Y} \in \ell^{p}$ and $S(\boldsymbol{f} ; \dot{\mathcal{P}})-\mathbf{Y}=\mathbf{0} \in \ell^{p}$. Then it can be defined

$$
\|S(\boldsymbol{f} ; \dot{\mathcal{P}})-\boldsymbol{Y}\|_{p}=\|\mathbf{0}\|_{p}=0
$$

or 


$$
\|S(\boldsymbol{f} ; \dot{\mathcal{P}})-\boldsymbol{Y}\|_{p}=\left\|\sum_{i=1}^{n}\left(x_{i}-x_{i-1}\right) \boldsymbol{f}\left(t_{i}\right)-\boldsymbol{Y}\right\|_{p}=\left(\sum_{k=1}^{\infty}\left|\sum_{i=1}^{n}\left(x_{i}-x_{i-1}\right) f_{k}\left(t_{i}\right)-Y_{k}\right|^{p}\right)^{\frac{1}{p}}
$$

From (4) and (7), obtained

$$
\|S(\boldsymbol{f} ; \dot{\mathcal{P}})-\boldsymbol{Y}\|_{p}=\left\|\sum_{i=1}^{n}\left(x_{i}-x_{i-1}\right) \boldsymbol{f}\left(t_{i}\right)-\boldsymbol{Y}\right\|_{p}=\left(\sum_{k=1}^{\infty}\left|\sum_{i=1}^{n}\left(x_{i}-x_{i-1}\right) f_{k}\left(t_{i}\right)-Y_{k}\right|^{p}\right)^{\frac{1}{p}}<\varepsilon
$$

Lemma 3.3 Let $\left(\ell^{p},\|\cdot\|_{p}\right)$ is a normed space. The sequence of functions $\boldsymbol{f}=\left(f_{k}\right):[a, b] \subset \mathbb{R} \rightarrow$ $\ell^{p}$ is said to be Riemann integrable on $[a, b] \subset \mathbb{R}$ if and only if each function

is Riemann integrable on $[a, b] \subset \mathbb{R}$.

$$
f_{k}:[a, b] \subset \mathbb{R} \rightarrow \mathbb{R}, k=1,2, \ldots, n, \ldots
$$

\section{Proof:}

It will show $\boldsymbol{f}=\left(f_{k}\right):[a, b] \subset \mathbb{R} \rightarrow \ell^{p}$ Riemann integrable on $[a, b] \subset \mathbb{R}$ if and only if each function $f_{k}:[a, b] \subset \mathbb{R} \rightarrow \mathbb{R}, k=1,2, \ldots$ is Riemann integrable on $[a, b] \subset \mathbb{R}$.

(i) $\Rightarrow$ Supposedly $\boldsymbol{f}=\left(f_{k}\right) \in \ell^{p}$ is Riemann intergble on $[a, b] \subset \mathbb{R}$, then according to Lemma 3.1.2, there is a sequence of functions $\boldsymbol{Y}=\left(Y_{k}\right) \in \ell^{p}$ with the characteristic that for each $\varepsilon>0$, there is $\delta>0$ such that

$$
\|S(\boldsymbol{f} ; \dot{\mathcal{P}})-\boldsymbol{Y}\|_{p}=\left(\sum_{k=1}^{\infty}\left|\sum_{i=1}^{n}\left(x_{i}-x_{i-1}\right) f_{\mathrm{k}}\left(t_{i}\right)-\mathrm{Y}_{\mathrm{k}}\right|^{p}\right)^{\frac{1}{p}}<\varepsilon
$$

for all partitions tagged $\dot{\mathcal{P}}$ of $[a, b] \subset \mathbb{R}$ with $\|\dot{\mathcal{P}}\|<\delta$.

From Inequation (8), for a real number $p$ with $1 \leq p<\infty$, it is obtained

$$
\left|\sum_{i=1}^{n}\left(x_{i}-x_{i-1}\right) f_{1}\left(t_{i}\right)-Y_{1}\right|^{p}+\left|\sum_{i=1}^{n}\left(x_{i}-x_{i-1}\right) f_{2}\left(t_{i}\right)-Y_{2}\right|^{p}+\cdots<\varepsilon^{p} .
$$

Because $\left|\sum_{i=1}^{n}\left(x_{i}-x_{i-1}\right) f_{\mathrm{k}}\left(t_{i}\right)-\mathrm{Y}_{\mathrm{k}}\right|^{p} \geq 0$, for each $k=1,2, \ldots$, it is specifically obtained

$$
\left|\sum_{i=1}^{n}\left(x_{i}-x_{i-1}\right) f_{1}\left(t_{i}\right)-Y_{1}\right|^{p}<\epsilon^{p}
$$

or for an arbitrary $\varepsilon>0$ obtained

$$
\left|\sum_{i=1}^{n}\left(x_{i}-x_{i-1}\right) f_{1}\left(t_{i}\right)-\mathrm{Y}_{1}\right|<\varepsilon
$$

Notice that from the definition of space $\ell^{p}, f_{1}$ is a real-valued function, which is $f_{1}:[a, b] \subset \mathbb{R} \rightarrow \mathbb{R}$ 


\section{Aswad Hariri Mangalaeng, Naimah Aris, Jusmawati Massalesse}

Based on Definition 2.1.5, Inequation (9) shows that the function $f_{1}$ are Riemann integrable on $[a, b] \subset \mathbb{R}$ for all partitions $\dot{\mathcal{P}}$ of $[a, b] \subset \mathbb{R}$ with $\|\dot{\mathcal{P}}\|<\delta$. The same thing applies to real functions $f_{k}:[a, b] \subset \mathbb{R} \rightarrow \mathbb{R}, k=2,3, \ldots$ Thus, it is obtained that each function $f_{k}, k=1,2, \ldots$ is Riemann integrable on $[a, b] \subset \mathbb{R}$ for all partitions $\dot{\mathcal{P}}$ of $[a, b] \subset \mathbb{R}$ with $\|\dot{\mathcal{P}}\|<\delta$.

Thus, it is evidenced that if the sequence of functions of $f=\left(f_{k}\right)$ is Riemann integrable on $[a, b] \subset \mathbb{R}$ then each function $f_{k}:[a, b] \subset \mathbb{R} \rightarrow \mathbb{R}, k=1,2, \ldots$ is Riemann integrable on $[a, b] \subset \mathbb{R}$.

(ii) $\quad \Leftrightarrow$ It is known that $\boldsymbol{f}=\left(f_{k}\right)$ is a sequence of real functions, then based on the proof by Lemma 3.1.2, it is obtained that if each function $f_{k}:[a, b] \subset \mathbb{R} \rightarrow \mathbb{R}, k=1,2, \ldots$ is Riemann Integrable on $[a, b] \subset \mathbb{R}$ then there is an sequence of functions of $\boldsymbol{Y}=\left(Y_{k}\right) \in$ $\ell^{p}$ with the properties that for every $\varepsilon>0$, there is $\delta>0$ such that

$$
\begin{aligned}
\|S(\boldsymbol{f} ; \dot{\mathcal{P}})-\boldsymbol{Y}\|_{p} & =\left\|\sum_{i=1}^{n}\left(x_{i}-x_{i-1}\right) \boldsymbol{f}\left(t_{i}\right)-\boldsymbol{Y}\right\|_{p} \\
& =\left(\sum_{k=1}^{\infty}\left|\sum_{i=1}^{n}\left(x_{i}-x_{i-1}\right) f_{k}\left(t_{i}\right)-Y_{k}\right|^{p}\right)^{\frac{1}{p}}<\varepsilon
\end{aligned}
$$

for every tagged partition $\dot{\mathcal{P}}$ of $[a, b] \subset \mathbb{R}$ with $\|\dot{\mathcal{P}}\|<\delta$. It means that the sequence of functions of $\boldsymbol{f}=\left(f_{k}\right):[a, b] \subset \mathbb{R} \rightarrow \ell^{p}$ is Riemann Integrable on $[a, b] \subset \mathbb{R}$.

From (i) and (ii), it can be concluded that the sequence of functions $\boldsymbol{f}=\left(f_{k}\right):[a, b] \subset \mathbb{R} \rightarrow \ell^{p}$ is Riemann integrable on $[a, b] \subset \mathbb{R}$ if and only if each function $f_{k}:[a, b] \subset \mathbb{R} \rightarrow \mathbb{R}, k=1,2, \ldots$ is Riemann integrable on $[a, b] \subset \mathbb{R}$.

As in the Riemann integral concept of real functions, the Riemann integral value of the sequence of functions in the normed space $\left(\ell^{p},\|\cdot\|_{p}\right)$ is also seen as the limit of Riemann sum. In full, given by Lemma 3.1.4 below.

Lemma 3.4 Let $\left(\ell^{p},\|\cdot\|_{p}\right)$ is a normed space. The sequence of functions $\boldsymbol{f}=\left(f_{k}\right):[a, b] \subset \mathbb{R} \rightarrow$ $\ell^{p}$ is said to be Riemann integrable on $[a, b] \subset \mathbb{R}$ if and only if

$$
\lim _{\|\dot{\mathcal{P}}\| \rightarrow 0} S(f ; \dot{\mathcal{P}})=\lim _{\|\dot{\mathcal{P}}\| \rightarrow 0} \sum_{i=1}^{n} \boldsymbol{f}\left(t_{i}\right)\left(x_{i}-x_{i-1}\right)=\boldsymbol{Y},
$$

for a sequence $\boldsymbol{Y}=\left(Y_{k}\right) \in \ell^{p}, k=1,2, \ldots$.

\section{Proof.}

(i) $\Rightarrow$ Supposedly the functions sequence $\boldsymbol{f}=\left(f_{k}\right) \in \ell^{p}$ is Riemann integrable on $[a, b] \subset \mathbb{R}$ then according to Lemma 3.1.3 we obtain $\boldsymbol{f}$ is Riemann integrable on $[a, b] \subset \mathbb{R}$ if and only if the functions $f_{k}:[a, b] \subset \mathbb{R} \rightarrow \mathbb{R}, k=1,2, \ldots$ are Riemann integrable on $[a, b] \subset$ $\mathbb{R}$. 


\section{Aswad Hariri Mangalaeng, Naimah Aris, Jusmawati Massalesse}

Since $f_{k}$ is a real function, then from Equation (1) we get $f_{k}$ are Riemann integrable on $[a, b] \subset \mathbb{R}$ if and only if

$$
\lim _{\|\dot{\mathcal{P}}\| \rightarrow 0} S\left(f_{\cdot_{k}} ; \dot{\mathcal{P}}\right)=Y_{k} \text { or } \lim _{\|\dot{\mathcal{P}}\| \rightarrow 0} \sum_{i=1}^{n}\left(x_{i}-x_{i-1}\right) f_{\cdot k}\left(t_{i}\right)=Y_{k},
$$

$Y_{k} \in \mathbb{R}, k=1,2, \ldots$

Because $\mathrm{S}(\boldsymbol{f} ; \dot{\mathcal{P}})=\sum_{i=1}^{n}\left(x_{i}-x_{i-1}\right) f\left(t_{i}\right)$ and $\|\dot{\mathcal{P}}\|$ is not a function of $S(\boldsymbol{f} ; \dot{\mathcal{P}})$, then obtained

$$
\begin{aligned}
\lim _{\|\dot{\mathcal{P}}\| \rightarrow 0} S(\boldsymbol{f} ; \dot{\mathcal{P}}) & =\lim _{\|\dot{\mathcal{P}}\| \rightarrow 0} \sum_{i=1}^{n}\left(x_{i}-x_{i-1}\right) \boldsymbol{f}\left(t_{i}\right) \\
& =\lim _{\|\dot{\mathcal{P}}\| \rightarrow 0} \sum_{i=1}^{n}\left(x_{i}-x_{i-1}\right)\left(f_{1}\left(t_{i}\right), f_{2}\left(t_{i}\right), \ldots\right) \\
& =\left(\lim _{\|\dot{\mathcal{P}}\| \rightarrow 0} \sum_{i=1}^{n}\left(x_{i}-x_{i-1}\right) f_{k}\left(t_{i}\right), \lim _{\|\dot{\mathcal{P}}\| \rightarrow 0} \sum_{i=1}^{n}\left(x_{i}-x_{i-1}\right) f_{k}\left(t_{i}\right), \ldots\right) \\
& =\left(Y_{1} . Y_{2}, \ldots\right) .
\end{aligned}
$$

Notice that $\boldsymbol{f} \in \ell^{p}$ and $\|\dot{\mathcal{P}}\|$ are not functions of $S(\boldsymbol{f} ; \dot{\mathcal{P}})$, so that based on the properties of the vector space $\ell^{p}$ is obtained $\lim _{\|\dot{\mathcal{P}}\| \rightarrow 0} S(\boldsymbol{f} ; \dot{\mathcal{P}}) \in \ell^{p}$, then if it is defined $\boldsymbol{Y}=$ $\left(Y_{1}, Y_{2}, \ldots\right)$ is obtained $\mathbf{Y} \in \ell^{p}$ and $\lim _{\|\dot{\mathcal{P}}\| \rightarrow 0} S(\boldsymbol{f} ; \dot{\mathcal{P}})=\mathbf{Y}$.

(ii) $\quad \Leftarrow$ On the other hand, if $\lim _{\|\dot{\mathcal{P}}\| \rightarrow 0} S(\boldsymbol{f} ; \dot{\mathcal{P}})=\mathbf{Y}$, for a $\boldsymbol{Y}=\left(Y_{1}, Y_{2}, \ldots\right) \in \ell^{p}$ then it is obtained $\lim _{\|\dot{\mathcal{P}}\| \rightarrow 0} S(\boldsymbol{f} ; \dot{\mathcal{P}}) \in \ell^{p}$ and

$$
\begin{gathered}
\lim _{\|\dot{\mathcal{P}}\| \rightarrow 0} S(\boldsymbol{f} ; \dot{\mathcal{P}})=\mathbf{Y} \\
\lim _{\|\dot{\mathcal{P}}\| \rightarrow 0} \sum_{i=1}^{n}\left(x_{i}-x_{i-1}\right) \boldsymbol{f}\left(t_{i}\right)=\mathbf{Y} \\
\lim _{\|\dot{\mathcal{P}}\| \rightarrow 0} \sum_{i=1}^{n}\left(x_{i}-x_{i-1}\right)\left(f_{1}\left(t_{i}\right), f_{2}\left(t_{i}\right), \ldots\right)=\left(Y_{1}, Y_{2}, \ldots\right) \\
\left(\lim _{\|\dot{\mathcal{P}}\| \rightarrow 0} \sum_{i=1}^{n}\left(x_{i}-x_{i-1}\right) f_{k}\left(t_{i}\right), \lim _{\|\dot{\mathcal{P}}\| \rightarrow 0} \sum_{i=1}^{n}\left(x_{i}-x_{i-1}\right) f_{k}\left(t_{i}\right), \ldots\right)=\left(Y_{1}, Y_{2}, \ldots\right) .
\end{gathered}
$$

So for each $k=1,2, \ldots$ it is obtained $\lim _{\|\dot{\mathcal{P}}\| \rightarrow 0} S\left(f_{\cdot_{k}} ; \dot{\mathcal{P}}\right) \in \mathbb{R}$ and $\lim _{\|\dot{\mathcal{P}}\| \rightarrow 0} S\left(f_{\cdot_{k}} ; \dot{\mathcal{P}}\right)=$ $Y_{k}$ or $\lim _{\|\dot{\mathcal{P}}\| \rightarrow 0} \sum_{i=1}^{n}\left(x_{i}-x_{i-1}\right) f_{\cdot k}\left(t_{i}\right)=Y_{k}$ which is Equation (10). From Equation (10) it is obtained $\boldsymbol{f}$ is Riemann integrable on $[a, b] \subset \mathbb{R}$.

From (i) and (ii), it can be concluded that the sequence of functions $\boldsymbol{f}=\left(f_{k}\right):[a, b] \subset \mathbb{R} \rightarrow \ell^{p}$ is Riemann integrable on $[a, b] \subset \mathbb{R}$ if and only if 


\section{Aswad Hariri Mangalaeng, Naimah Aris, Jusmawati Massalesse}

$$
\lim _{\|\dot{\mathcal{P}}\| \rightarrow 0} S(\boldsymbol{f} ; \dot{\mathcal{P}})=\lim _{\|\dot{\mathcal{P}}\| \rightarrow 0} \sum_{i=1}^{n} f\left(t_{i}\right)\left(x_{i}-x_{i-1}\right)=\mathbf{Y},
$$

for a sequence $\mathbf{Y}=\left(\mathrm{Y}_{k}\right) \in \ell^{p}, k=1,2, \ldots$

Lemma 3.5 Let $\left(\ell^{p},\|\cdot\|_{p}\right)$ is a normed space. If the sequence of functions of $\boldsymbol{f}=\left(f_{k}\right):[a, b] \subset$ $\mathbb{R} \rightarrow \ell^{p}$ is Riemann integral in $[a, b] \subset \mathbb{R}$, then the Riemann integral value of $\boldsymbol{f}$ is unique.

\section{Proof:}

Supposedly $\boldsymbol{Y}=\left(Y_{k}\right) \in \ell^{\mathrm{p}}$ and $\boldsymbol{Y}^{\prime}=\left(Y_{k}^{\prime}\right) \in \ell^{\mathrm{p}}$ are Riemann integral values of the sequence of function $\boldsymbol{f}=\left(f_{k}\right) \in \ell^{\mathrm{p}}$ in $[a, b]$, means that for each $\varepsilon>0$, there is $\delta>0$ such that

$$
\|S(\boldsymbol{f} ; \dot{\mathcal{P}})-\boldsymbol{Y}\|_{p}<\frac{\varepsilon}{2} \text { and }\left\|S(\boldsymbol{f} ; \dot{\mathcal{P}})-\mathbf{Y}^{\prime}\right\|_{p}<\frac{\varepsilon}{2}
$$

for all tagged partitions $\dot{\mathcal{P}}$ of $[a, b] \in \mathbb{R}$ with $\|\dot{\mathcal{P}}\|<\delta$. By using some properties of the norm are obtained

$$
\begin{aligned}
\left\|\boldsymbol{Y}-\boldsymbol{Y}^{\prime}\right\|_{p} & =\left\|\boldsymbol{Y}-S(\boldsymbol{f} ; \dot{\mathcal{P}})+S(\boldsymbol{f} ; \dot{\mathcal{P}})-\boldsymbol{Y}^{\prime}\right\|_{p} \\
& =\|\boldsymbol{Y}-S(\boldsymbol{f} ; \dot{\mathcal{P}})\|_{p}+\left\|\boldsymbol{S}(\boldsymbol{f} ; \dot{\mathcal{P}})-\boldsymbol{Y}^{\prime}\right\|_{p} \\
& =\|S(\boldsymbol{f} ; \dot{\mathcal{P}})-\boldsymbol{Y}\|_{p}+\left\|S(\boldsymbol{f} ; \dot{\mathcal{P}})-\boldsymbol{Y}^{\prime}\right\|_{p} \\
& <\frac{\varepsilon}{2}+\frac{\varepsilon}{2}=\varepsilon .
\end{aligned}
$$

It is obtained $\left\|\boldsymbol{Y}-\boldsymbol{Y}^{\prime}\right\|_{p}=0$ or $\boldsymbol{Y}=\boldsymbol{Y}^{\prime}$. This means that each Riemann integral value of $\boldsymbol{f}$ is always same. Thus, it is concluded that the Riemann integral value of the sequence of functions $\boldsymbol{f}=\left(f_{k}\right):[a, b] \subset \mathbb{R} \rightarrow \ell^{\mathrm{p}}$ is unique.

\section{Conclusion}

A Riemann sum of the sequence of functions $\boldsymbol{f}=\left(f_{k}\right):[a, b] \subset \mathbb{R} \rightarrow \ell^{p}$ over the tagged partition $\dot{\mathcal{P}}$ of $[a, b] \subset \mathbb{R}$ is $S(\boldsymbol{f} ; \dot{\mathcal{P}})=\sum_{i=1}^{n}\left(x_{i}-x_{i-1}\right) f\left(t_{i}\right)$, whose proof can be seen in Lemma 3.1. The conditions of $\boldsymbol{f}$ to be Reimann Integral can be obtained, namely:

1. The sequence of functions $\boldsymbol{f}=\left(f_{k}\right):[a, b] \subset \mathbb{R} \rightarrow \ell^{p}$ is said to be Riemann integrable on $[a, b] \subset \mathbb{R}$ if there is a $\boldsymbol{Y}=\left(Y_{k}\right) \in \ell^{p}$ with the property that for each $\varepsilon>0$ there is $\delta>0$ such that

$$
\begin{aligned}
\|S(f ; \grave{\mathcal{P}})-\boldsymbol{Y}\|_{p} & \\
& =\left\|\sum_{i=1}^{n} \boldsymbol{f}\left(t_{i}\right)\left(x_{i}-x_{i-1}\right)-\boldsymbol{Y}\right\|_{p}=\left(\sum_{k=1}^{\infty}\left|\sum_{i=1}^{n}\left(x_{i}-x_{i-1}\right) f_{k}\left(t_{i}\right)-Y_{k}\right|^{p}\right)^{\frac{1}{p}} \\
& <\varepsilon
\end{aligned}
$$

for all partitions tagged $\dot{\mathcal{P}}$ of $[a, b] \subset \mathbb{R}$ with $\|\dot{\mathcal{P}}\|<\delta$,

2. The sequence of functions $\boldsymbol{f}=\left(f_{k}\right):[a, b] \subset \mathbb{R} \rightarrow \ell^{p}$ is said to be Riemann integrable on $[a, b] \subset \mathbb{R}$ if and only if each function $f_{k}:[a, b] \subset \mathbb{R} \rightarrow \mathbb{R}, k=1.2, \ldots$ is Riemann integrable on $[a, b] \subset \mathbb{R}$, and

3. The sequence of functions $\boldsymbol{f}=\left(f_{k}\right):[a, b] \subset$ ] $\rightarrow \ell^{p}$ is said to be Riemann integrable on $[a, b] \subset \mathbb{R}$ if and only if $\lim _{\|\dot{\mathcal{P}}\| \rightarrow 0} S(\boldsymbol{f} ; \dot{\mathcal{P}})=\lim _{\|\dot{\mathcal{P}}\| \rightarrow 0} \sum_{i=1}^{n} \boldsymbol{f}\left(t_{i}\right)\left(x_{i}-x_{i-1}\right)=\boldsymbol{Y}$, for a $\boldsymbol{Y}=\left(Y_{k}\right) \in \ell^{p}, k=1.2, \ldots$ 
Aswad Hariri Mangalaeng, Naimah Aris, Jusmawati Massalesse

From these conditions, it is obtained that the Reimann integral value of the sequence of functions $\boldsymbol{f}$ is unique that has been proven in Lemma 3.5.

\section{References}

[1] Aji, P. (2016). Integral Riemann Bernilai Barisan $\ell^{1}$. Skripsi. Bandar Lampung: Universitas Lampung.

[2] Bartle, R. G., \& Sherbert, D. R. (2011). Introduction to Real Analysis, 4th Edition. United States of America: John Wiley \& Sons, Inc.

[3] Chernysh, E. (2018). The Riemann Integral For Functions Mapping To Banach Spaces. Departmen of Mathematics, McGill University.

[4] Kreyzig, E. (1978). Introductory Functional Analysis with Application. Canada: John Wiley \& Sons, Inc. 